\title{
LA POSTHUMANIDAD COMO UN BIEN OBJETIVO: LOS PELIGROS DEL FUTURISMO EN EL DEBATE SOBRE LA OPTIMIZACIÓN GENÉTICA HUMANA
}

Andrés Vaccari ${ }^{1}$

Resumen: La figura de la posthumanidad es invocada en una amplia gama de argumentos que defienden el atractivo o incluso el imperativo moral del perfeccionamiento humano por medio de la ingeniería genética. La posthumanidad se presenta en estos argumentos como un estado altamente beneficioso, el punto final de un proceso de evolución dirigida al que debemos abocar nuestros esfuerzos. Centrándome en los escritos del pensador transhumanista Nick Bostrom, cuestiono este rol normativo de la posthumanidad como justificación del proyecto de una eugenesia liberal. Para empezar, argumento que los costos y beneficios de un estado posthumano son imposibles de calcular. Por otro lado, identifico ciertos problemas relacionados con la posicionalidad e instrumentalidad de las capacidades aumentadas. Estas capacidades son valuables en la medida en que (1) proveen una ventaja relativa a un contexto y (2) son instrumentales al futuro bienestar de las personas. Argumento que no se puede establecer una conexión necesaria entre capacidades y bienestar y, por lo tanto, la promesa de la posthumanidad como un estado beneficioso es difícil de tomar seriamente.

Palabras clave: ingeniería genética, tecnologías de optimización, optimización genética, teoría ética

Post humanity as an objective good: the dangers of futurism in the debate about enhancement of human genome

\begin{abstract}
The figure of posthumanity is invoked in a wide range of arguments that advertise the appeal or even the moral imperative of human enhancement through means of genetic engineering. Posthumanity is presented in these arguments as a highly beneficial state, the end point of a process of directed evolution to which we must devote our efforts. Focusing on the writings of transhumanist philosopher Nick Bostrom, in this article I question this normative role of posthumanity as a legitimation for liberal eugenics. For a start, I argue that the costs and benefits of a posthuman state are impossible to calculate. On the other hand, I identify certain problems concerning the positionality and instrumentality of augmented capacities. These capacities can be considered valuable inasmuch as they (1) provide an advantage relative to a context and (2) they are instrumental to the future benefit of persons. I argue that we cannot establish a necessary connection between capacities and wellbeing, thus the promise of posthumanity as a beneficial state is difficult to take seriously.
\end{abstract}

Key words: genetic engineering, enhancement technologies, genetic enhancement, ethical theory

A pós-humanidade como um bem objetivo: os perigos do futurismo no debate sobre a otimizaçáo genética humana

Resumo: A figura da pós-humanidade é invocada em uma ampla gama de argumentos que defendem o atrativo, ou inclusive, o imperativo moral do aperfeiçoamento humano por meio da engenharia genética. A pós-humanidade apresenta-se nestes argumentos como um estado altamente beneficioso, o ponto final de um processo de evoluçáo dirigida, para o qual devemos envidar os nossos esforços. Centrando-me nos escritos do pensador transhumanista Nick Bostrom, questiono este papel normativo da pós-humanidade como justificativa do projeto de uma eugenia liberal. Para começar, argumento que os custos e benefícios de um estado pós-humano são impossíveis de calcular. Por outro lado, identifico certos problemas relacionados com a posicionalidade e instrumentalidade das capacidades aumentadas. Estas capacidades são avaliáveis na medida em que (1) apresentem uma vantagem relativa a um contexto e (2) são instrumentos para o futuro bem-estar das pessoas. Argumento que não se pode estabelecer uma conexão necessária entre capacidades e bem-estar e, portanto, a promessa da pós-humanidade como um estado beneficioso é difícil de ser admitida seriamente.

Palavras-chave: engenharia genética, tecnologias de otimização, otimização genética, teoria ética

\footnotetext{
${ }^{1}$ Coordinador, Programa de la Filosofía de la Tecnología, Fundación Bariloche, Argentina Correspondencia: Andres.Vaccari@mq.edu.au
} 


\section{Transhumanismo y evolución dirigida}

El distinguido biólogo inglés Julian Huxley fue el primero en acuñar el término "transhumanismo" en 1957:

"La especie humana puede, si lo desea, trascender en sí, no solo de forma esporádica (un individuo aquí de una manera, otra persona allí de otra manera), pero en su totalidad, como humanidad. Necesitamos un nombre para esta nueva creencia. Quizás nos servirá el nombre de transhumanismo: el hombre sigue siendo hombre, pero se trasciende a sí mismo, realizando nuevas posibilidades de y para su naturaleza humana(1:13).

El transhumanismo es un movimiento filosófico que promulga el advenimiento de un futuro estado de la humanidad, denominado "posthumanidad". Es un futuro en el que la humanidad habrá intervenido racionalmente en su propia evolución para reinventarse de acuerdo con sus propios sueños y aspiraciones, transcendiendo así la prerrogativa natural de su existencia y realizando su pleno potencial. Es también, como asevera Huxley, un estado colectivo, lo cual implica que estos nuevos poderes y virtudes de la posthumanidad estarán distribuidos equitativamente o de un modo que beneficie a una proporción lo suficientemente amplia de la humanidad. Nick Bostrom, el pensador transhumanista más destacado en la actualidad, asevera:

"En última instancia, es posible que estas optimizaciones puedan hacernos a nosotros o a nuestros descendientes, 'posthumanos', seres con una longevidad indefinida, facultades intelectuales mucho mayores que las de cualquier ser humano actual (y tal vez sensibilidades o modalidades completamente nuevas), así como la capacidad de controlar sus propias emociones" (2:203).

El debate sobre la optimización humana (buman enhancement) abarca una amplia gama de tecnologías, que van desde productos farmacéuticos e implantes neuronales hasta las intervenciones genéticas. Sin embargo, hay una clase de tecnologías que ameritan un análisis aparte. Me refiero a la ingeniería de la línea germinal, la cual promete (o amenaza con) introducir cambios permanentes y trasmisibles al patrimonio genómico de la huma- nidad. Hasta ahora no ha sido ensayada en humanos, pero parece ser una cuestión de tiempo. Los escenarios posthumanos en su mayoría se basan en este tipo de intervención genética, la cual tiene el objeto de producir cambios a la especie en el nivel de su genética.

El andamiaje central de estos argumentos es la idea de una humanidad mejorada, compuesta por seres con capacidades intelectuales, físicas y emocionales muy superiores a los de la humanidad actual. Esta narrativa recibe comúnmente el nombre de Evolución Dirigida (Directed Evolution, or Rationally Guided Evolution). John Harris presenta este escenario de la siguiente manera:

"Hemos llegado a un punto en la historia humana en el que nuevos intentos de hacer del mundo un lugar mejor tendrán que incluir no solo cambios en el mundo, sino cambios a la humanidad (...) Propongo la sabiduría y la necesidad de intervenir (...) tomando el control de la evolución y de nuestro desarrollo futuro hasta el punto (de hecho, más allá del punto) donde nosotros los humanos nos hemos transformado, tal vez, en una especie totalmente nueva $y$, sin duda, una mejor" $(3: 3-4)$.

La Evolución Dirigida es el tipo más ambicioso de argumento en pro de la optimización genética humana. Mientras la mayoría de los argumentos que examinan esta cuestión se limitan a tratar ciertos problemas circunscritos y de alcance más inmediato (tales como las responsabilidades y deberes de los padres, o las políticas que deberían ser adoptadas por el sistema de salud pública), el transhumanismo apuesta a un salto fenomenológico mucho más arriesgado. Cabe aclarar que este tipo de narrativa no se encuentra exclusivamente en la literatura transhumanista y es una tesis muy difundida en la promoción de la ingeniería genética. Aunque gran parte del transhumanismo sea difícil de tomar en serio, sus principios centrales se encuentran — como ya lo ha señalado Francis Fukuyama- "implícitos en gran parte de la agenda de investigación de la biomedicina contemporánea"(4). Esta amplia difusión es una de las razones por las que debemos examinar críticamente estos argumentos. La propuesta de la Evolución Dirigida proyecta los efectos de la reprogenética a un futuro indefinido, en el que 
modificaciones al genoma de la especie afectarán a toda la humanidad directa o indirectamente. Pero el rasgo más significativo de este argumento es que nos pide que aceptemos a la posthumanidad como un bien, un estado más benéfico para la humanidad, al cual debemos abocar nuestros esfuerzos. O sea que la posthumanidad se aduce como una razón por la cual debemos aceptar las biotecnologías de optimización y promover su extensa distribución.

Antes de continuar, debemos aclarar que el debate sobre la optimización humana parte de una distinción entre intervención terapéutica y optimización que ha suscitado una gran controversia(5-8). En general, se admite que cierta gama de intervenciones terapéuticas (tales como la eliminación de condiciones determinadas genéticamente, por ejemplo, el Síndrome de Down y la fibrosis cística) no plantea problemas éticos de esta índole, dado que se enmarca más claramente dentro de los fines terapéuticos tradicionales de la medicina. No cabe duda de que, en muchos casos, ciertas tecnologías (genéticas, la nanotecnología, los medios farmacéuticos, la biónica y otras tantas) puedan significar un gran beneficio para la salud y la calidad de vida de las personas. Pero la optimización no es una cuestión tan simple de resolver, porque propone la posibilidad de modificar organismos sanos con el objeto de incrementar ciertas capacidades, tales como la memoria, la visión, el sistema inmunológico, las propensiones a ciertos tipos de personalidad, e incluso la duración de la vida. Entonces, los proponentes de la optimización deben recurrir a otros tipos de argumentos, típicamente basados en una idea de beneficio. Aquí me ocuparé de examinar un tipo de argumento que aboga por los beneficios de este tipo de intervenciones biotecnológicas, basadas en un cierto tipo de razones, las cuales juzgo desacertadas.

Partiré de un supuesto central: para juzgar si un curso de acción es deseable o imperioso debemos primero decidir si dicha acción conducirá a un bien que podamos conocer o calcular de algún modo. El argumento que nos ocupa trata de persuadirnos de que la posthumanidad es una razón por la cual debemos liberar estas nuevas tecnologías al mercado. Sin embargo, argumentaré que la posthumanidad no es calculable y por lo tanto no puede ser tomada como una razón para aceptar estas tecnologías en principio. Mi argumento se estructura del siguiente modo:

En la sección siguiente, identifico el modelo de acceso a los bienes usualmente pregonado por los transhumanistas. Este modelo se basa en nociones liberales del sujeto autónomo y racional, las cuales se hallan usualmente acompañadas por una defensa de la lógica del mercado libre. Identifico una teoría deliberativa de los valores como el eje central del argumento transhumanista. Argumento que existe una insalvable contradicción entre estados emergentes del mercado — caracterizados por una pluralidad de valores- y la idea de un bien calculable y lo suficientemente generalizado. En particular, exploro los escollos que nos impiden calcular este futuro estado benéfico.

En la Sección 3 identifico dos áreas claves que dificultan aún más el cálculo de beneficios y, por ende, el riesgo moral implicado en estas tecnologías. Examino primero la posicionalidad de las capacidades y luego discuto la relación entre capacidad y virtud, y entre capacidad y beneficio. Mi conclusión es que no hay una relación necesaria entre capacidades aumentadas y beneficios resultantes.

La conclusión examina un último argumento de Bostrom: la posthumanidad como bien objetivo. $\mathrm{Mi}$ argumento concluye con ciertas observaciones prescriptivas acerca de los límites de la especulación moral en el debate sobre la optimización humana.

\section{Libertades, modelos y universalidad}

El transhumanismo ha optado por un modelo de implementación claramente individualista en el que la libertad de elección es la potestad moral del individuo autónomo. Para situar este debate en su contexto más amplio, unos de los problemas bioéticos en torno a la modificación genética humana concierne los modelos de implementación. Buchanan et al.(5:11-13) distinguen dos polos hacia los cuales gravitan diferentes perspectivas éticas sobre este tema: el modelo de Salud Pública y el modelo de Servicio Personal. El modelo de Salud Pública enfatiza el análisis consecuencialista de costos y beneficios en grupos humanos, eva- 
luando la implementación de cualquier tecnología biomédica dada en términos de provechos y daños agregados. El modelo de Servicio Personal, por el contrario, se basa en la elección privada y argumenta que el uso de nuevas tecnologías genéticas debe ser consignado al criterio de la autonomía individual. Podríamos también alinear con estas dos tendencias la oposición entre dos modelos eugenésicos: el político-institucional y el liberal-economicista(9:116).

Los argumentos transhumanistas gravitan hacia esta segunda posición. Hay quienes abogan por un marco de completa desregulación o regulación muy limitada(10-12), y otros que argumentan que el acceso a estas tecnologías debe ser cuidadosamente regulado(13). Sin embargo, existe una clara discrepancia entre el modelo de libre mercado y la idea de una posthumanidad como un marco heurístico y moral definido. Por un lado, el transhumanismo trata de seducirnos con la imagen de un estado posthumano incitante, cuya consumación es concebida como un imperativo moral. Escribe Bostrom: "Cada día que se demora la introducción de la efectiva optimización genética humana es un día de potencial cultural e individual perdido, y un día de tormento para los que sufren enfermedades que podrían haber sido prevenidas" (14:499). Por otro lado, estas propuestas se comprometen con un modelo deliberativo de los valores, en el cual este futuro se concibe como el resultado de un agregado de incontables decisiones individuales. De este modo, el modelo de mercado libre restringe la soberanía moral sobre decisiones reproductivas a los padres, quienes son los que decidirán las características de su progenie.

Esta visión deliberativa de la moral está fundada sobre el modelo del yo autónomo liberal (J. S. Mill e Immanuel Kant son los filósofos claves en esta tradición). Sin embargo, Bostrom hace una distinción entre libertades morfológicas y libertades reproductivas(2:203). Las primeras abarcan intervenciones tecnológicas que el individuo elige aplicarse a sí mismo, es decir, las libertades individuales sobre el cuerpo y la mente propios. Las segundas, las libertades reproductivas, abarcan decisiones sobre las características genéticas de los descendientes de la persona. Ahora, mientras las libertades morfológicas se encuentran (de acuer- do a este argumento) dentro de la esfera soberana del yo autónomo, las reproductivas imponen un límite evidente al alcance filosófico del libertarismo, dado que son acciones que afectan a otros. Es decir, a futuros otros. La ingeniería de línea germinal, tanto como el tipo de libertades implicadas en la narrativa de la evolución dirigida, están comprendidas en esta última categoría.

"Una democracia liberal debería normalmente permitir incursiones en las libertades morfológicas solo en los casos en que alguien está abusando de estas libertades para dañar a otra persona" (2:210). Pero Bostrom también argumenta que el enfoque libertario no es adecuado en el caso de las modificaciones de la línea germinal. Se debe adoptar un enfoque cuidadosamente regulatorio, que limite ciertas libertades de los padres al tiempo que distribuya equitativamente las opciones de optimización disponibles(14:499-500). Bostrom también aconseja la adopción de políticas sociales que mitiguen las tendencias a la creciente desigualdad que acarrean estas tecnologías(14:503). Por último, Bostrom destaca la importancia de promover la mejora de características que tengan "externalidades positivas": rasgos que derivan en un bien social y no un bien puramente individual.

Esto quiere decir que argumentos a favor de la evolución dirigida deben, en última instancia, basarse en concepciones del bien común, promulgando el "enorme potencial para usos profundamente valiosos y beneficiosos para la humanidad" implicado en el proyecto de Evolución Dirigi$\mathrm{da}(2: 203)$.

Este bien común puede concebirse de dos maneras: como un bien colectivo (de una sociedad o grupo de individuos considerados en el contexto de sus interrelaciones e instituciones, y considerando desigualdades sociales y otros factores) o bien como una suma del bienestar personal de personas consideradas individualmente. Pero ni Bostrom ni ningún transhumanista deja en claro cuál sería el mínimo número de personas necesario para obtener un estado más beneficioso. Tampoco queda claro cuál es el criterio por el cual evaluaríamos estos beneficios, dado que Bostrom se enfoca exclusivamente en el bienestar individual: "el valor que tiene una vida para la persona que la vive: qué tan bueno (o malo) es para el sujeto 
tener esta vida" (15). Bostrom, como hemos visto, hace referencia a bienes colaterales (externalidades), lo que implica que una vida también puede tener valor en términos de sus consecuencias para las vidas de otros y de la sociedad en general. Sin embargo, podemos apreciar que un argumento de este estilo necesitaría precisar qué tipo de externalidades serían deseables y cómo calcularíamos su beneficio colectivo.

Cualquiera sea el caso, deberíamos asegurarnos que estos cambios son lo suficientemente extendidos a lo largo y ancho de la población como para incidir significativamente en el bien común. Y aquí nos encontramos con el primer escollo. Los transhumanistas tratan de salvar la distancia entre estos dos aspectos de su argumento (la posthumanidad como bien común versus la posthumanidad como resultado agregado de decisiones individuales) por medio de una apelación a valores que parecen ser universales o autoevidentes. En 1998, el famoso biólogo molecular James Watson, premio Nobel y codescubridor del ADN, resumió el planteo de este modo: "Nadie tiene realmente las agallas para decirlo, pero si pudiésemos hacer mejores seres humanos sabiendo cómo agregar genes, ¿por qué no deberíamos hacerlo?" (16:12). Lo interesante de esta proposición es que, por medio de una pregunta retórica, supone que todos estamos de acuerdo en lo que constituye un humano "mejor" y que sería ridículo oponerse a algo que es mejor. Como nos dice Hauskeller, por definición, "sugerir que una optimización es mala es una contradicción de términos”(17:113).

Sin embargo, la certeza implícita en este tipo de suposición se desvanece una vez que analizamos la cuestión más detenidamente. ¿¿Por qué deberíamos hacerlo? ¿Hay realmente un consenso acerca de cuáles normas guiarían la construcción de "mejores" seres humanos?

El problema principal concierne el alcance y naturaleza de estos cambios. Desde Darwin, sabemos que la humanidad es una especie biológica que solo puede ser definida históricamente como el resultado de sucesivos cambios adquiridos y variaciones acumulativas. Se sigue de esto que las naturalezas de las diferentes especies son fenómenos estadísticos que se refieren a la distribución "normal" de características en una población dada. En otras palabras, la naturaleza humana es un concepto poblacional. Como escribe Tim Lewens, "la única noción biológicamente respetable de la naturaleza humana que nos queda es una extremadamente permisiva, que enumera las disposiciones confiables de la especie humana en su totalidad. Esta concepción no ofrece una guía ética en el debate sobre la optimización y, de hecho, nos lleva a la conclusión de que alteraciones a la naturaleza humana son comunes en la historia de la especie" $(18: 460)^{2}$.

En este marco, el argumento de la evolución dirigida exige que concibamos a la posthumanidad en estos mismos términos poblacionales. ¿Estará esta posthumanidad definida por una distribución "confiable" de características, la cual nos permitiría hablar de una posthumanidad de rasgos estables o al menos identificables? Y, ¿cómo evaluaríamos esta distribución como más beneficiosa que la presente? La distribución de características tales como la altura, la inteligencia y la resistencia inmunológica es diversa en la actualidad. En definitiva, si una parte considerable de la población mundial decidiese crear una nueva generación de posthumanos, no queda claro cómo esto conduciría a un cambio medible en la distribución normal de estas características. Incluso menos definibles son los beneficios que acarrearía tamańo cambio fenotípico. O bien los cambios deben producirse al nivel de poblaciones enteras y alterar la distribución normal de las características de las mismas (el modelo político-institucional de la eugenesia clásica), o bien debemos aceptar el modelo de mercado claramente favorecido por el transhumanismo. El asunto aquí es que la futura forma de la posthumanidad sería determinada por valores diversos. Russell Powell sostiene que la diversidad de valores presente en las culturas humanas evitará que la posthumanidad se convierta en una especie de monocultivo que reduzca la capacidad adaptativa de la especie. Los individuos y las culturas no harán un uso común de las tecnologías de ingeniería genética, porque no hay concepciones valorativas comunes que se mantengan a través de las personas y las culturas:

"Es absurdo pensar que existe algo así como un

${ }^{2}$ Véase también Daniels(19) y la respuesta de Ramsey: "Características humanas, no obstante, no están distribuidas al azar en el set colectivo de las historias de vida humanas"(20:483). 
consenso sobre el valor y contenido de las complejas disposiciones humanas (como el gusto estético, el atractivo sexual o la virtud moral). Aunque hay ciertos principios de organización que son estables en todas las culturas (tales como la simetría morfológica), estos representan atolones en medio de un mar de valores diferentes para personas diferentes. Incluso si hay un acceso generalizado a las tecnologías de ingeniería genética, la disparidad de preferencias culturales, económicas, religiosas, morales, políticas y de otra índole, evitará la fijación de un pequeño subconjunto de fenotipos. De hecho, al permitir a la gente actuar sobre estas preferencias divergentes, estas tecnologías en realidad podrían incrementar la diversidad biológica humana, lo que permitiría nuevas (y de otra manera inaccesibles) combinaciones de características deseadas(21:213-214).

En este contexto, es instructivo evaluar el argumento de Ryuichi Ida, quien afirma que las culturas de Japón, y de otros países asiáticos influenciados por el budismo y el confucianismo, no tienden a compartir los valores de la biomedicina occidental sustancialmente implícitos en las nuevas tecnologías(22). Por lo tanto, la recepción de las ideas del transhumanismo es mayormente negativa en estas culturas. Y podemos extender este razonamiento a otras culturas que se mantienen al margen del universo de valores de la tecnociencia occidental moderna en el cual se enmarca el argumento transhumanista. Incluso en el caso de América Latina, el Caribe y España, podemos apreciar que estas ideas no han tenido una penetración cultural fuerte ${ }^{3}$.

Bostrom asume que el atractivo de su programa de valores transhumanistas es universal, y que cualquier persona racional asentiría a estos valores si deliberase lo suficiente: "la idea es que si examinamos nuestros valores cuidadosamente, encontraremos que incluyen valores cuya realización completa requeriría la posesión de capacidades posthumanas" (24:5). Hemos visto cómo "nuestros" valores no se hallan distribuidos de un modo lo suficientemente confiable como para precisar un grupo definido de capacidades posthumanas al que asentir como un fin deseado. Los escritos en

\footnotetext{
${ }^{3}$ Obviamente debemos reconocer variaciones significativas en las opiniones dentro de una misma cultura y que no hay una perspectiva unificada(23:12-13).
}

pro de la posthumanidad insisten en usar un truco retórico (el uso de la segunda persona plural) para ocultar lo que, en última instancia, es una perspectiva cultural localizada y específica. ¿Quiénes somos "nosotros"? De acuerdo a Bostrom, este "nosotros" se compone de personas de clase media de países industrializados occidentales, con un cierto poder adquisitivo y nivel de educación:

"Leemos y escribimos, usamos ropa, vivimos en ciudades, ganamos dinero y compramos comida en el supermercado, llamamos a la gente por teléfono, vemos la televisión, leemos el diario, conducimos coches, pagamos impuestos, votamos en las elecciones nacionales, mujeres dan a luz en los hospitales, la esperanza de vida es tres veces más que en el Pleistoceno, sabemos que la Tierra es redonda y que las estrellas son grandes nubes de gas iluminadas desde adentro por la fusión nuclear, y que el universo tiene aproximadamente 13,7 mil millones de ańos y es enormemente grande. A los ojos de un cazador-recolector, nosotros ya podríamos parecer "posthumanos" (2:213).

Esta estrategia retórica se halla muy extendida en argumentos a favor de estas tecnologías. En definitiva, esta estrategia sirve para crear un consenso artificial e incluso para ocultar posibles injusticias y desigualdades. Bostrom nos insta a asentir a la posthumanidad como un escenario deseado, un resultado, fin o logro que justifica el proyecto de la eugenesia liberal. Sin embargo, como hemos visto, este escenario no es consistente con la diversidad de valores y metas en vista de los cuales estas nuevas tecnologías serían utilizadas.

\section{Capacidades, virtudes y beneficios}

Me ocuparé aquí de examinar dos objeciones centradas en lo que podríamos llamar la ontología de las capacidades: las brechas entre capacidad, ejercicio virtuoso de dicha capacidad, y beneficio.

Primero, debemos enfatizar el hecho de que los beneficios colectivos de las características modificadas solo pueden computarse en referencia a un marco público que es sensible a la competitividad. Como argumentan Buchanan et al., la ventaja de ciertas características (por ejemplo, tener una mayor altura o una memoria fotográfica) siempre se plantea en un contexto en el que dichas caracte- 
rísticas se hallan distribuidas de un cierto modo "normal". Si todos pudiéramos incrementar nuestra altura unos centímetros, esto ya no sería una ventaja para nosotros. Se sigue que la distribución generalizada de ciertas modificaciones (denominadas "posicionales" debido a su beneficio relativo) sería contraproducente; o, peor, nos llevaría a una situación en que muchos otros se sentirían forzados a adoptar estas intervenciones incluso cuando originalmente no veían ningún beneficio en ellas(5:184-186). Esto último podría concebirse como un atropello a la autonomía de las personas que eligen no hacer uso de estas tecnologías. Se sigue de todo esto que no podemos calcular los beneficios de capacidades incrementadas de acuerdo con un marco estático de valores, dado que la introducción de dichas capacidades tiene el efecto de modificarlo.

El segundo problema es que el transhumanismo establece una relación automática y natural entre capacidades individuales y beneficios (sean individuales o colectivos). Pero, de hecho, toda concepción de estas capacidades solo puede ser instrumental. El valor máximo aquí es el bienestar del individuo (o de la suma de individuos) y las capacidades posthumanas son valuables solo en la medida en que posibilitan una existencia de mayor bienestar ${ }^{4}$. Sin embargo, el carácter instrumental de las capacidades posthumanas no garantiza la virtud ni el bienestar, porque no hay una relación necesaria entre capacidad y bienestar. Mis padres pueden haberme diseñado para ser un genio matemático, sin embargo yo prefiero pasar el día tomando cerveza y viendo telenovelas. Esto sería una vida beneficiosa de acuerdo a mis valores, pero no justificaría la decisión de mis padres. O podría yo haber elegido ser un genio matemático pero, por algún motivo, ser infeliz y estar constantemente deprimido. En el primer caso se nos plantea una brecha entre capacidad

${ }^{4}$ Dejaré de lado aquí otro problema muy serio, que concierne al efecto que una función o capacidad pueda tener en el contexto de la economía biológica, mental o emocional del individuo (o, más generalmente, su fenotipo). Mecanismos neurológicos y hormonales generalmente están causalmente ligados a una enorme multitud de funciones. Por ejemplo, Neil Levy cita los siguientes casos: la serotonina afecta la tendencia a la afiliación social, la respiración, la regulación cardiovascular, el apetito y la sensibilidad al dolor, entre otras funciones. La gente que tiene buena memoria tiene problemas con el pensamiento abstracto, aparentemente porque recordar detalles interfiere con el foco en los aspectos significativos de una situación(25:600). y valor, y en el segundo la brecha se halla entre capacidad y bienestar. Los tres (capacidad, valor y bienestar) no mantienen una relación suficientemente consistente como para fundar la idea de que la modificación de capacidades es beneficiosa por sí. Bostrom reconoce este problema y replica que "estas adscripciones de valor afirman que el objeto o propiedad normalmente hace una contribución positiva a lo que sea que tenga valor básico" (15). Sin embargo, esto es difícil de asegurar. Como bien argumentan Buchanan et al., debemos ser recelosos de cualquier intervención (sea genética o ambiental) que aumente una disposición que es meramente necesaria para una virtud, porque las capacidades son componentes y no constituyen la virtud en sí misma(5:180-1). La noción de virtud es necesaria en este contexto para precisar la ejecución virtuosa de una capacidad; es decir, una ejecución que conduzca al bien, tanto en términos de bienestar personal como de bienestar social o intersubjetivo; esta ejecución de la capacidad también justificaría la intención originaria que llevó a la modificación. Buchanan et al. ilustran este problema con el ejemplo imaginario de "Cynthia", una mujer con un alto grado de empatía e inteligencia emocional, que podría ser una asistente social pero es, en cambio, una estafadora que vende propiedades inexistentes a jubilados. Las capacidades de Cynthia claramente no conllevan a un bien, incluso cuando la práctica de la estafa sea beneficiosa para Cynthia.

Estas brechas entre diferentes aspectos ontológicos de la intervención (capacidad, valor, bienestar, virtud, contexto social e intersubjetivo, etc.) son éticamente significativas porque amplían el riesgo moral de las decisiones en torno a las modificaciones humanas. Estas objeciones en sí mismas no constituyen un argumento contra de la modificación genética humana, sino que ponen en relieve los problemas asociados a la valuación de sus beneficios; en particular, aquellos que surgen a raíz de las complejas interacciones causales entre las varias dimensiones implicadas.

\section{Conclusión: los espacios del ser}

En suma, podemos ver que los argumentos que presentan a la posthumanidad como un bien autoevidente no pueden ser aceptados tan temerariamente. Esto nos lleva a la siguiente conclusión: 
el debate sobre la optimización humana debería atenerse a áreas más concretas, tales como los modelos de salud, o las responsabilidades de padres hacia hijos. La idea de la posthumanidad puede servir como un marco heurístico en el cual discutir las posibilidades y peligros de las nuevas biotecnologías, pero no puede tener un rol moral o constituirse en una razón por la cual aceptar estas mismas tecnologías. Por ejemplo, el foco ético principal en lo concerniente a tecnologías de línea germinal parece centrarse en los padres, dado que ellos decidirían, en última instancia, qué hijos prefieren tener y cuáles modificaciones efectuar a su propia herencia genética. Gregory Stock argumenta que los padres muy probablemente decidan hacer intervenciones modestas(16:139). De acuerdo con Stock, los padres decidirán, y el futuro de la biología humana debe dejarse en las manos de las leyes del mercado y de decisiones individuales5. Podemos no estar de acuerdo con esta posición, pero su mérito principal es enfocar el debate sobre un área mucho más definida de competencia y autonomía moral. Es muy improbable (más allá de un grupo reducido de fervientes transhumanistas) que futuros padres decidan modificar a sus hijos en vista a crear una raza posthumana.

En este contexto cabe mencionar uno de los argumentos más ambiciosos de Bostrom en pro de la posthumanidad $(14,15,28)$, consistente en una visión de "valores posthumanos" que están más allá de nuestro alcance: "la manera correcta de favorecer a los seres humanos es permitirnos realizar nuestros ideales de un modo mejor y (...) algunos de nuestros ideales podrían estar fuera del espacio existencial (outside the space of modes of being) accesibles a nosotros con nuestra constitución biológica presente"(14:495).

Bostrom argumenta que deberíamos desarrollar nuestras capacidades en vista a explorar dimensiones valorativas más allá de nuestro entendimiento. "La gama de pensamientos, sentimientos, experiencias y actividades accesibles a los organismos humanos constituyen solo una parte minúscula de lo que es posible" (28). En suma, nuestro espacio existencial (determinado por nuestras capa-

${ }^{5}$ Julian Savulescu es un bioeticista famoso por esgrimir un argumento similar: el de la "beneficencia procreativa" (procreative beneficience) $(26,27)$. cidades biológicas y cognitivas) no nos permite apreciar el beneficio acarreado por las modificaciones que nos llevarán a la posthumanidad. Sería como preguntarle a un chimpancé si le gustaría convertirse en un humano. De hecho, Bostrom dice que existe la misma brecha entre las capacidades proyectivas de chimpancés y humanos que las que existen entre los humanos y posthumanos.

Nicholas Agar presenta una fuerte objeción a este argumento por medio de una analogía. Los perros tienen una gama olfativa más amplia que la de los humanos, lo cual les permite disfrutar el olor a carne podrida. Supongamos que posthumanos con una gama olfativa similar encuentren el olor a carne podrida igualmente deleitable: no se podría deducir de esto que debamos aceptar este tipo de valores(29:16).

Este argumento es interesante en la medida en que cuestiona el supuesto que ha guiado nuestra investigación; es decir, que no podemos asentir a una razón que no conocemos. Sin embargo, está claro que esta idea de la posthumanidad como bien objetivo no nos lleva muy lejos. Incluso parece violar el principio de autonomía, obligándonos a aceptar un bien que no podemos conocer.

Si estamos preparados a aceptar la noción de la optimización humana como una empresa moralmente aceptable (un punto en el que no hay un claro consenso y que es un foco de ardiente debate), entonces la bioética debería bajar a la tierra y plantearse preguntas más definidas y abiertas a corroboración empírica: ¿cuáles capacidades hemos de mejorar y de qué modo? ¿Podemos construir un marco ético común a todas estas nuevas tecnologías? ¿Quiénes se beneficiarán? ¿Quiénes decidirán? ¿Cuáles son los contextos culturales y políticos en los que se adoptarán estas tecnologías? (23:3). De otro modo, corremos el riesgo de presentar como realidades lo que, al fin y al cabo, son nada más que fantasías. 


\section{Referencias}

1. Huxley J. New bottles for new wine. London: Chatto \& Windus; 1957.

2. Bostrom N. In defense of posthuman dignity. Bioethics 2005; 19(3): 202-214.

3. Harris J. Enhancing evolution: The ethical case for making better people. Princeton (NJ): Princeton University Press; 2007.

4. Fukuyama F. Transhumanism. Foreign Policy 2004. Available from: http://www.foreignpolicy.com/articles/2004/09/01/ $\underline{\text { transhumanism }}$

5. Buchanan A, Brock DW, Daniels N, Wikler D. From chance to choice: Genetics and justice. Cambridge University Press; 2000.

6. Resnik DB. The Moral Significance of the Therapy-Enhancement Distinction in Human Genetics. Cambridge Quarterly of Healthcare Ethics 2000; 9(3): 365-377.

7. Colleton L. The elusive line between enhancement and therapy and its effects on health care in the U.S. Journal of Evolution \& Technology 2008; 18(1): 70-78.

8. Lin P. Therapy and Enhancement: Is There a Moral Difference? Drawing a Principled Line between the Two Is Complicated, if It Even Exists. Point of View 2009; 29(3). Available from: http://www.genengnews.com/gen-articles/therapyand-enhancement-is-there-a-moral-difference/2959/

9. Bello Reguera G. El riesgo moral: Los límites de la vida humana y la democratización de la ética. CTS: Revista Iberoamericana de Ciencia, Tecnología y Sociedad, Selección 2012; 111-125.

10. Transhumanist Declaration (2002). Available from: http://humanityplus.org/philosophy/transhumanist-declaration/

11. Bailey R. Liberation biology: The scientific and moral case for the biotech revolution. NY: Prometheus Books; 2005.

12. Naam R. More than human: Embracing the promise of biological enhancement. NY: Broadway Books; 2005.

13. Lin P, Allhoff F. Against unrestricted human enhancement. Journal of Evolution \& Technology 2008; 18(1): 35-41.

14. Bostrom N. Human genetic enhancements: A transhumanist perspective. The Journal of Value Inquiry 2004; 37: 493506.

15. Bostrom N. Why I want to be a posthuman when I grow up. 2008. Available from: http://www.nickbostrom.com/posthuman.pdf Accessed 08/07/09.

16. Stock G. Redesigning Humans: Our Inevitable Genetic Future. New York: Houghton Mifflin; 2002.

17. Hauskeller M. Cognitive enhancement: To what end? Cognitive Enhancement: Trends in Augmentation of Human Performance 2013; 1: 113-123.

18. Lewens T. Human nature: The very idea. Philosophy and Technology 2012; 25: 459-474.

19. Daniels N. Can anyone really be talking about ethically modifying human nature? In Bostrom N, Savulescu J, eds. Human Enhancement. New York: Oxford University Press; 2009: 25-42.

20. Ramsey G. How Human nature can inform enhancement: a Commentary on Tim Lewens's Human nature: the very Idea. Philosophy and Technology 2012; 25: 479-483.

21. Powell R. The Evolutionary Biological Implications of Human Genetic Engineering. Journal of Medicine and Philosophy 2010; 37(1): 204-226.

22. Ida R. Should we improve human nature? An interrogation from an Asian perspective. In Bostrom N, Savulescu J, eds. Human Enhancement. New York: Oxford University Press; 2009.

23. Bostrom N, Savulescu J. Human enhancement ethics: The state of the debate. In Bostrom N, Savulescu J, eds. Human Enhancement. New York: Oxford University Press; 2009.

24. Bostrom N. Human vs. Posthuman: Letter to the editor. The Hastings Center Report 2007; 37(5): 4-5.

25. Levy N. Ecological engineering: reshaping our environments to achieve our goals. Philosophy and Technology 2012; 25: 589-604.

26. Savulescu J. Procreative beneficience: Why we should select the best children. Bioethics 2001; 15(5/6): 413-426.

27. Savulescu J, Kahane G. The moral obligation to create children with the best chance of the best life. Bioethics 2009; 23(5): 274-290.

28. Bostrom N. Transhumanist values. 2003. Available from: http://www.nickbostrom.com/ethics/values.html

29. Agar N. Human vs. Posthuman. Hastings Center Report 2007; 37(5): 4-7. 